\title{
Testing QCD with hypothetical tau leptons
}

\author{
S. J. Brodsky, J. R. Peláez, ${ }^{*}$ and N. Toumbas \\ Stanford Linear Accelerator Center, Stanford University, Stanford, California 94309
}

(Received 21 October 1998; published 17 June 1999)

\begin{abstract}
We construct new phenomenological tests of perturbative QCD by considering a hypothetical $\tau$ lepton of arbitrary mass which decays hadronically through the electromagnetic current. Its hadronic branching ratio can be computed directly as an integral over the $e^{+} e^{-}$annihilation cross section ratio, $R_{e^{+}} e^{-}$. More generally, we can design a set of commensurate scale relations which test the applicability and self-consistency of leading twist QCD predictions by varying the weight function away from the form associated with the $V-A$ decay of the physical $\tau$. This method allows the wide range of $R_{e^{+} e^{-}}$data (or other similar observables which define an effective charge) to be used as renormalization scheme and scale invariant probes of QCD.
\end{abstract}

[S0556-2821(99)05713-6]

PACS number(s): 12.38.Qk, 12.38.Bx, 13.65.+i, 14.60.Fg

The $\tau$ lepton hadronic width is potentially one of the most important sources for the high precision determination of the QCD coupling $\alpha \overline{M S}[1,2]$. The perturbative QCD (PQCD) analysis of the $\tau$ width has been refined by constructing moments of hadronic decay distributions which minimize sensitivity to the low energy part of the hadronic spectrum [3]. However, it is still uncertain whether the $\tau$ mass is high enough to trust PQCD, mainly due to the distortion of hadronic final states [4].

In this paper we construct new renormalization scale- and scheme-independent tests of PQCD which can be applied to any observable which defines an effective charge. These selfconsistency tests are motivated by the relation between the hadronic width of a hypothetical $\tau$ lepton of arbitrary mass which decays hadronically through the vector current to a specific integral over the measured annihilation ratio $R_{e^{+} e^{-}}$ $=\sigma\left(e^{+} e^{-} \rightarrow\right.$ hadrons $) / \sigma\left(e^{+} e^{-} \rightarrow \mu^{+} \mu^{-}\right)$. Such hypothetical $\tau$ leptons, with masses $M<M_{\tau}$, have already been considered in Ref. [3]. The hypothetical character of these leptons allows us to generalize these tests to a more general class of physical observables and to generalized functional moments. As we shall show, these relations are fundamental properties of QCD which severely test the applicability and reliability of leading-twist perturbative predictions.

Quantum field-theoretic predictions which relate physical observables cannot depend on theoretical conventions such as the renormalization scheme or scale. The most wellknown example is the "generalized Crewther relation" [5] in which the leading twist PQCD corrections to the Bjorken sum rule at a given lepton momentum transfer $Q^{2}$ are inverse to the QCD corrections to $R_{e^{+} e^{-}}$at a corresponding c.m. energy squared, $s^{*}=s^{*}\left(Q^{2}\right)$, independent of the choice of renormalization scheme. The ratio of the scales $s^{*} / Q^{2}$ has been computed to next leading order (NLO) in PQCD. Such leading-twist predictions between observables are called "commensurate scale relations"' and are identical for conformal and nonconformal theories [6].

Another important example of commensurate scale relations is the connection between the all-orders leading-twist

\footnotetext{
*On leave of absence from the Departamento de Física Teórica, Universidad Complutense, 28040 Madrid, Spain.
}

PQCD corrections to the $\tau$ lepton's width ratio, $R_{\tau}=\Gamma\left(\tau^{-}\right.$ $\rightarrow \nu_{\tau}+$ hadrons $) / \Gamma\left(\tau^{-} \rightarrow \nu_{\tau} e^{-} \bar{\nu}_{e}\right)$, and those for $R_{e^{+} e^{-}}$. Assuming for now $f$ massless flavors, PQCD yields

$$
R_{e^{+} e^{-}}(\sqrt{s})=\left(3 \sum_{f} q_{f}^{2}\right)\left[1+\frac{\alpha_{R}(\sqrt{s})}{\pi}\right],
$$

where $\alpha_{R}$ can be written as a series in $\alpha_{s} / \pi$ in any given renormalization scheme. Note that $\alpha_{R}$ is an effective charge [7] because it satisfies the Gell-Mann-Low renormalization group equation with the same coefficients $\beta_{0}$ and $\beta_{1}$ as the usual coupling $\alpha_{s}$. Similarly, we can define an effective charge $\alpha_{\tau}$ as follows:

$$
R_{\tau}\left(M_{\tau}\right)=R_{\tau}^{0}\left(M_{\tau}\right)\left[1+\alpha_{\tau}\left(M_{\tau}\right) / \pi\right]
$$

Leading-twist QCD predicts $\alpha_{\tau}\left(M_{\tau}\right)=\alpha_{R}\left(\sqrt{s^{*}}\right)$ to all orders in perturbation theory. The ratio of the commensurate scales is known in NLO PQCD:

$$
\sqrt{s^{*}} / M_{\tau}=\exp \left\{-\frac{19}{24}-\frac{169}{128}\left[\alpha_{R}\left(M_{\tau}\right) / \pi\right]+\cdots\right\} .
$$

This result was originally obtained in [6] by using NNLO predictions for $\alpha_{R}$ and $\alpha_{\tau}$ obtained in the modified minimal subtraction ( $\overline{\mathrm{MS}}$ ) scheme and eliminating $\alpha \overline{M S}$. However, as we shall show here, the QCD prediction for $\sqrt{s^{*}} / M_{\tau}$ also follows from the fact that both effective charges evolve with universal $\beta_{0}$ and $\beta_{1}$ coefficients. The fact that $R_{\tau}$ can be written as

$$
\begin{aligned}
R_{\tau}\left(M_{\tau}\right)= & \frac{2}{\left(\sum_{f} q_{f}^{2}\right)} \int_{0}^{M_{\tau}^{2}} \frac{d s}{M_{\tau}^{2}}\left(1-\frac{s}{M_{\tau}^{2}}\right)^{2}\left(1+\frac{2 s}{M_{\tau}^{2}}\right) \\
& \times R_{e^{+} e^{-}}(\sqrt{s})
\end{aligned}
$$

implies, by the mean value theorem, that $\alpha_{R}$ and $\alpha_{\tau}$ are related by a scale shift. [We have used $\left|V_{u d}\right|^{2}+\left|V_{u s}\right|^{2}=1$, as in [8]. Notice that in order to include NNLO corrections in $\alpha_{\tau}$, we must modify the $O\left(\alpha_{s}^{3}\right)$ coefficient of $\alpha_{R}$ by setting $\left(\sum_{f} q_{f}\right)^{2}=0$.] However, the prediction for the ratio $\sqrt{s^{*}} / M_{\tau}$ in Eq. (3) is a specific property of PQCD.

An empirical test of the commensurate relation, Eq. (3), is problematic since the real $\tau$ lepton mass seems uncomfortably low for the applicability of leading-twist QCD predictions. However, we can construct more general tests of PQCD by considering a hypothetical $\tau$ lepton of arbitrary 
mass $M$ which decays hadronically through the vector current. Its hadronic width ratio is given by a specific integral over the measured $R_{e}{ }^{+} e^{-}$. More generally, we can design a set of commensurate scale relations and PQCD tests by varying the weight function away from the form associated with the $V-A$ decay. In fact, this method can be applied to functional moments of any observable which defines an effective charge as a function of a Mandelstam variable.

In this paper we shall apply the new commensurate scale relations to generalized moments integrated over a large range of available $R_{e^{+} e^{-}}$data. In order to test the leadingtwist PQCD predictions, it will be important to take into account the specific scale-breaking effects attributable to the $s \bar{s}, c \bar{c}, b \bar{b}$ quark thresholds. Also, following [9], we shall smear the annihilation data in order to regulate resonances and other distortions due to final state interactions. By smearing $R_{e^{+} e^{-}}$over a range of energy, $\Delta E$, we focus the physics to the time $\Delta t=1 / \Delta E$ where an analysis in terms of PQCD quark and gluon subprocesses is appropriate. Thus this method can also be interpreted as a test of duality. Scheme-independent relations between $R_{e^{+}} e^{-}$and $\tau$ decay have also been recently discussed in [2].

Given an observable $O(s)$, and its associated effective charge $\alpha_{O}$, we can define new effective charges as follows:

$\alpha_{f}(M) \equiv \int_{0}^{M^{2}} \frac{d s}{M^{2}} f\left(\frac{s}{M^{2}}\right) \alpha_{O}(\sqrt{s}) / \int_{0}^{M^{2}} \frac{d s}{M^{2}} f\left(\frac{s}{M^{2}}\right)$.

We can choose $f(x)$ to be any smooth, integrable function of $x=s / M^{2}$. [For the particular choice, $f_{\tau}(x)=(1-x)^{2}(1$ $+2 x)$ and $O=R_{e^{+} e^{-}}, \alpha_{O}^{f_{\tau}}=\alpha_{R}^{f_{\tau}}$ is simply $\alpha_{\tau}$.] The mean value theorem then implies $\alpha_{f}(M)=\alpha_{O}\left(\sqrt{s_{f}^{*}}\right), \quad 0 \leqslant s_{f}^{*}$ $\leqslant M^{2}$. Note that this relation only involves data for the observable $O(s)$ and thus provides an interesting selfconsistency test for the applicability of leading twist QCD. Dimensional analysis ensures that $\sqrt{s_{f}^{*}}=\lambda_{f} M$, where $\lambda_{f}$ possibly depends on $\alpha_{O}$. To obtain an estimate for $\lambda_{f}$ we consider the running of $\alpha_{O}$ up to third order:

$$
\begin{gathered}
\frac{\alpha_{O}(\sqrt{s})}{\pi}=\frac{\alpha_{O}(M)}{\pi}-\frac{\beta_{0}}{4} \ln \left(\frac{s}{M^{2}}\right)\left(\frac{\alpha_{O}(M)}{\pi}\right)^{2} \\
+\frac{1}{16}\left[\beta_{0}^{2} \ln ^{2}\left(\frac{s}{M^{2}}\right)-\beta_{1} \ln \left(\frac{s}{M^{2}}\right)\right]\left(\frac{\alpha_{O}(M)}{\pi}\right)^{3} \ldots .
\end{gathered}
$$

We substitute for $\alpha_{O}$ in Eq. (5) to find

$$
\begin{aligned}
\frac{\alpha_{f}(M)}{\pi}= & \frac{\alpha_{O}(M)}{\pi}-\frac{\beta_{0}}{4}\left(\frac{I_{1}}{I_{0}}\right)\left(\frac{\alpha_{0}(M)}{\pi}\right)^{2} \\
& +\frac{1}{16}\left[\beta_{0}^{2}\left(\frac{I_{2}}{I_{0}}\right)-\beta_{1}\left(\frac{I_{1}}{I_{0}}\right)\right]\left(\frac{\alpha_{O}(M)}{\pi}\right)^{3} \ldots,
\end{aligned}
$$

where $I_{l}=\int_{0}^{1} f(x)(\ln x)^{l} d x$. By setting $s=s^{*}$ in Eq. (6) and comparing with Eq. (7), we extract

$$
\lambda_{f}=\exp \left\{\frac{I_{1}}{2 I_{0}}+\frac{\beta_{0}}{8}\left[\left(\frac{I_{1}}{I_{0}}\right)^{2}-\frac{I_{2}}{I_{0}}\right] \frac{\alpha_{O}(M)}{\pi}\right\} .
$$

Note that if $f(x)$ is positive on the interval $[0,1]$, then $I_{1} / I_{0}$ is negative as expected. Using $f_{\tau}(x)$, Eq. (8) is nothing but
Eq. (3). Also, since $\lambda_{f}$ is a constant to leading order, $\alpha_{f}$ should satisfy the same renormalization group (RG) equation as $\alpha_{O}$ with the same coefficients $\beta_{0}$ and $\beta_{1}$. In other words, $\alpha_{f}$ is an effective charge.

As an example we will apply this self-consistency test to the $O=R_{e^{+} e^{-}}$data, using different weight functions $f(x)$ and varying $M$ to see whether we obtain the PQCD behavior. In general, the weight function $f(x)$ should be chosen to suppress the low energy region, where non-perturbative effects are important. Thus in the following, we shall set $f(x)=x^{k}$, where $k$ is some positive number. In such a case, we have

$$
\alpha_{k}(M)=\alpha_{R}\left(\lambda_{k} M\right) \quad \text { with } \lambda_{k}=e^{I_{1 k} / 2 I_{0 k}},
$$

where $I_{1 k}=\int_{0}^{1} x^{k} \ln x d x$ and $I_{0 k}=\int_{0}^{1} x^{k} d x$. Note that as $\mathrm{k}$ increases, $I_{1 k} / 2 I_{0 k} \rightarrow 0$, and therefore $\sqrt{s^{*}} \rightarrow M$. For very large $k$, we lose sensitivity to the details of PQCD. It is particularly interesting to use such a test to probe the energy region close to the $\tau$ mass $M_{\tau}$.

The main difficulty in comparing with $R_{e^{+} e^{-}}$data is that we can no longer consider massless flavors and that we observe hadrons instead of quarks. Following [9] the effect of quark masses can be approximately taken into account if we use

$$
\begin{aligned}
R_{e^{+} e^{-}}(\sqrt{s}) & =3 \sum_{1}^{f} q_{i}^{2} \frac{v_{i}\left(3-v_{i}^{2}\right)}{2}\left[1+g\left(v_{i}\right) \frac{\alpha_{R}(\sqrt{s})}{\pi}\right] \\
\equiv & R_{0}(\sqrt{s})+R_{S c h}(\sqrt{s}) \frac{\alpha_{R}(\sqrt{s})}{\pi} \\
g(v) & =\frac{4 \pi}{3}\left[\frac{\pi}{2 v}-\frac{3+v}{4}\left(\frac{\pi}{2}-\frac{3}{4 \pi}\right)\right]
\end{aligned}
$$

where $v_{i}=\sqrt{1-4 m_{i}^{2} / s}$ is the velocity of the initial quarks in their c.m. frame. The $v_{i}\left(3-v_{i}^{2}\right) / 2$ factor is the parton model mass dependence and $g(v)$ is a QCD modification [10] of the Schwinger correction [11]. The quark masses have been taken as effective parameters in order to provide a good fit to the smeared data. In principle, all these corrections spoil the relation in Eq. (9). However these factors are only relevant close to the corresponding quark thresholds. At higher energies the correction factors tend to unity and the precise values of the quark masses become irrelevant. Our main analysis will be restricted to this regime, although it could be possible to extend it by relating the quark masses directly to those of the resonances [12]. Nevertheless, we still cannot compare directly with the data since there is no direct correspondence between quark and hadronic thresholds. To obtain a meaningful comparison we have to smear both the PQCD results and the data. Following [9] we define smeared quantities as follows:

$$
\bar{R}(\sqrt{s})=\frac{\Delta}{\pi} \int_{0}^{\infty} \frac{R\left(\sqrt{s^{\prime}}\right)}{\left(s-s^{\prime}\right)^{2}-\Delta^{2}} d s^{\prime} .
$$

Note that in the $\Delta \rightarrow 0$ limit, we recover the original quantity. In what follows we use the standard value $\Delta=3 \mathrm{GeV}^{2}$ $[9,13]$. The smearing effect can be seen by comparing Fig. 1, which shows an interpolation of the $R_{e^{+} e^{-}}$data [14], with Fig. 2. For completeness, we also include in Fig. 2 the smeared results from NLO PQCD and from the naive parton model $\left(\alpha_{R}=0\right)$. 


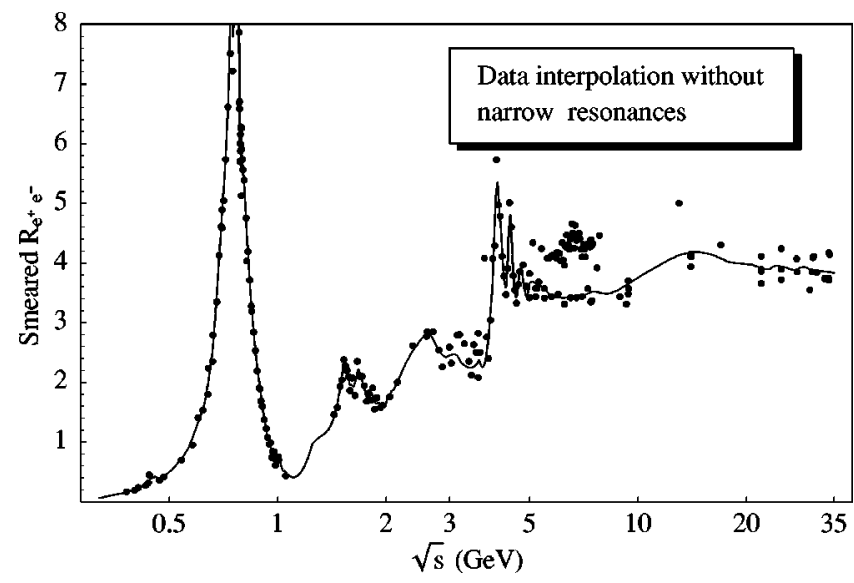

FIG. 1. Interpolation of the central values of $R_{e^{+} e^{-}}$data [14]. Narrow resonances are taken into account using their Breit-Wigner form. Note that there is a discrepancy in the central values of experiments between 5 and $10 \mathrm{GeV}$, that above $20 \mathrm{GeV}$ we have two or three clearly different central values at the same $\sqrt{s}$, and that the point at $13 \mathrm{GeV}$ is much higher than other nearby data.

In order to integrate over $R_{e^{+} e^{-}}$, we need to interpolate, but not fit, the data. Notice that any fit using the QCD functional dependence will always satisfy the commensurate scale relations, even if its quality is poor. To avoid this bias, we have interpolated the central values of the data by means of " $r$-term simple moving averages" up to $30 \mathrm{GeV}$ (to avoid electroweak contributions). That is, if we have a series of raw data $z_{1}, \ldots z_{n}$, we obtain the new set of smoothed data $\sum_{j=0}^{r-1} w_{j} z_{t-j}$ for $t=r, \ldots n$, with $\sum_{j=0}^{r-1} w_{j}=1$. We have used $r$ ranging from 2 to 6 for different energy regions, and our moving averages are "simple" because all the weights $w_{j}$ are equal. Finally, the resulting smoothed data have been interpolated using cubic-splines. In addition, the narrow resonances that do not appear in Fig. 1 are parametrized using the Breit-Wigner formula.

We have thus eliminated the QCD biases up to $30 \mathrm{GeV}$. Above that energy we have matched a logarithmic function whose functional dependence is inspired by QCD, but its contribution in the smearing integrals is negligible for small $\sqrt{s}$.

Unfortunately we cannot extract directly the effective charges from their corresponding smeared ratios since they

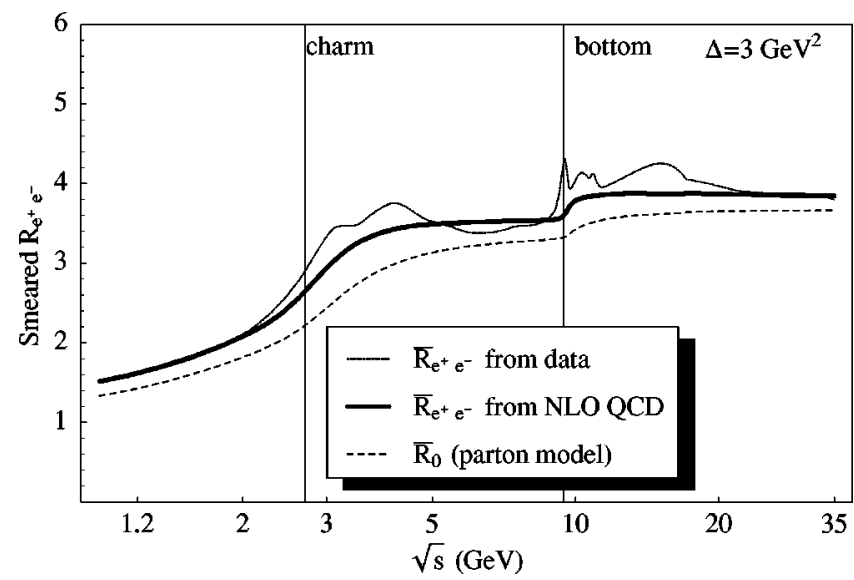

FIG. 2. Effect of smearing on $R_{e^{+} e^{-}}$.

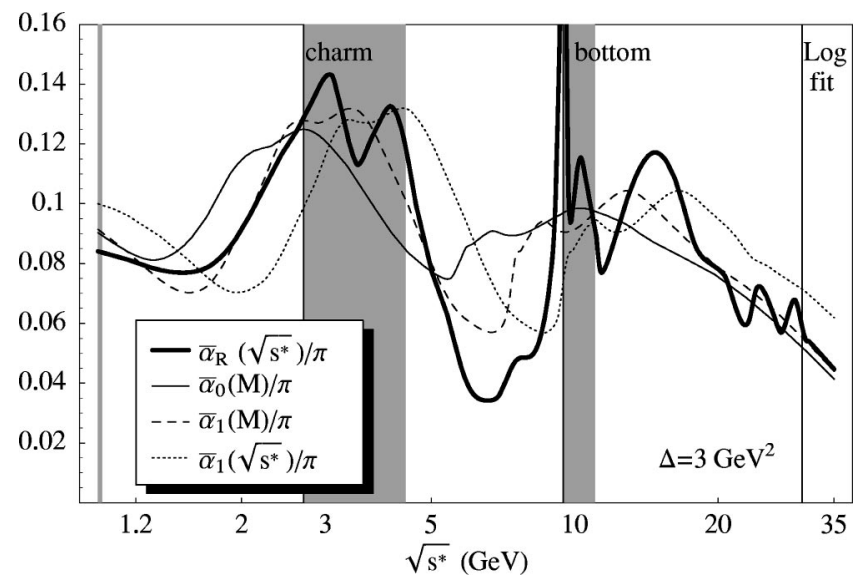

FIG. 3. Comparison between $\bar{\alpha}_{R}\left(\sqrt{s^{*}}\right)$ and different $\bar{\alpha}_{k}$ moments at $M=\sqrt{s^{*}} / \lambda_{k}$. The dotted line shows how the agreement is spoiled if we do not shift $\sqrt{s^{*}}$ to $M$.

are multiplied by other functions inside the smearing integral. However, using Eqs. (10) and (12), we define smeared charges:

$$
\bar{\alpha}_{R}(\sqrt{s})=\left[\bar{R}_{e^{+} e^{-}}(\sqrt{s})-\bar{R}_{0}(\sqrt{s})\right] / \bar{R}_{S c h}(\sqrt{s})
$$

and similarly for $\bar{\alpha}_{k}$. In the massless $\Delta \rightarrow 0$ limit we recover the standard effective charges. We expect the smeared charges to satisfy Eq. (8) in energy regions where the threshold corrections can be neglected.

In Fig. 3 we compare $\bar{\alpha}_{R}$ at $\sqrt{s^{*}}$ with $\bar{\alpha}_{k}$ moments at $M$ $=\sqrt{s^{*}} / \lambda_{k}$. The agreement for $\alpha_{0}$ is poor since the low energy region is not sufficiently suppressed. However we find a reasonable agreement for $\alpha_{1}$ in several regions; we also show how this agreement disappears if we do not shift the argument of $\alpha_{1}$ from $\sqrt{s^{*}}$ to $M=\sqrt{s^{*}} / \lambda_{1}$. Above $30 \mathrm{GeV}$ the commensurate scale relations are satisfied almost identically, which is not surprising since above that energy we have fitted with a QCD-inspired behavior. From $15 \mathrm{GeV}$ up to $30 \mathrm{GeV}$ different experiments have measured rather different central values at very similar, or even the same, energies. The smooth interpolation of these points produces artificial oscillations around the mean values of the data. Since these oscillations are centered on the $\alpha_{k}$ curves, the agree-

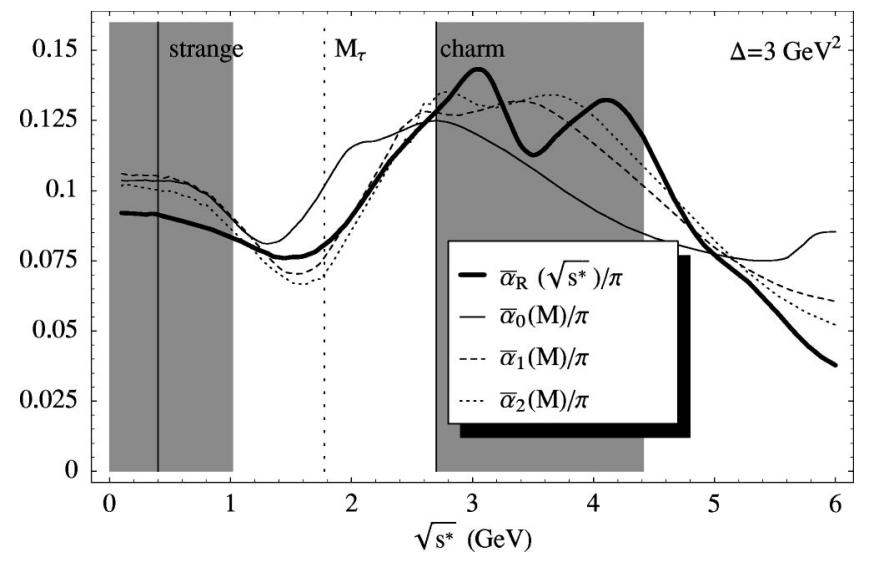

FIG. 4. Comparison between $\bar{\alpha}_{R}\left(\sqrt{s^{*}}\right)$ and different $\bar{\alpha}_{k}$ moments at $M=\sqrt{s^{*}} / \lambda_{k}$ in the low energy region. 
ment is reasonable, given the quality of the data. In the region between 5 and $10 \mathrm{GeV}$, there seems to be some controversy about the compatibility between different experiments (see Fig. 1 and Ref. [15]). It has become standard not to use the older cross section data points (which are high) as we have done in Fig. 1. Although the most recent data are compatible within their experimental errors with QCD expectations, their central values are systematically lower, which is why Eq. (9) does not seem to hold. Given more accurate data, the tests we are proposing, together with a thorough error analysis, will shed light on this situation.

The low energy region is shown in more detail in Fig. 4. Taking into account that we are only using LO QCD and central data values, the agreement between the shaded regions looks quite satisfactory. This is encouraging for the real $\tau$ lepton, which sits in a region where PQCD results may be applicable since it is primarily sensitive to the light $u, d, s$ flavors. Nevertheless, by looking at energies $\sqrt{s} \sim 1.5 \mathrm{GeV}$, our results seem to support the claims that the $R_{e^{+} e^{-}}$data could be $6-7 \%$ lower than expected from $R_{\tau}$ data [2].

Motivated by the commensurate scale relations connecting the moments of the lepton hadronic decay spectrum to $R_{e^{+} e^{-}}$, we have derived basic scheme-independent tests of leading-twist PQCD which depend only on the universal terms of the $\beta$ function. By employing generalized weight functions, we have obtained relations of an observable to itself at a different scale. We have emphasized that these relations are self-consistency tests which in principle can be applied to any observable which defines an effective charge. It is not necessary to have a relation to another observable as in the case of $R_{\tau}$ and $R_{e^{+} e^{-}}$. We have seen, however, that a direct application to timelike observables such as $R_{e^{+} e^{-}}$is complicated by the distortions of narrow and broad resonances, the physical effects of the quark pair thresholds, and the imprecision of much of the data. Smearing the data over an energy range helps, but does not totally remove, the effects due to final-state interactions. Quark threshold distortions are partially alleviated by using the Schwinger corrections at small velocity, but the domain of non-relativistic velocity introduces its own complications, including sensitivity of the QCD running coupling to the soft $\alpha_{s} m_{q}$ scale [16]. Nevertheless, these self-consistency tests still yield relevant information, such as support for the claims that the measurements of the annihilation cross section may be inaccurate in certain energy regions. On the positive side, our analysis indicates that the mass range of the physical $\tau$ lepton is potentially clear of large quark mass corrections since it is well below the $c \bar{c}$ threshold. Higher precision measurements of $R_{e^{+} e^{-}}$throughout the energy domain below the $Z^{0}$ boson are very much needed in order to definitively test the new commensurate relations derived here. Further applications to other observables are in progress.

We are indebted to M. Swartz for assistance with the experimental data. J.R.P. thanks the Spanish Ministerio de Educación y Cultura for financial support, and the SLAC Theory Group for their hospitality. This research was partially supported by the U.S. Department of Energy under contract DE-AC03-76SF00515 and the Spanish CICYT under contract AEN97-1693.
[1] E. Braaten, Phys. Rev. Lett. 60, 1606 (1988); Phys. Rev. D 39, 1458 (1989); E. Braaten, S. Narison, and A. Pich, Nucl. Phys. B373, 581 (1992).

[2] S. Groote et al., Phys. Rev. Lett. 79, 2763 (1997).

[3] F. Le Diberder and A. Pich, Phys. Lett. B 289, 165 (1992); S. Narison and A. Pich, ibid. 304, 359 (1993); S. Narison, ibid. 358, 113 (1995); 361, 121 (1995); M. Girone and M. Neubert, Phys. Rev. Lett. 76, 3061 (1996); S. Groote, J. G. Korner, and A. A. Pivovarov, Phys. Lett. B 407, 66 (1997); M. Davier, Phys. Rev. D 58, 096014 (1998); R. Barate et al., Eur. Phys. J. C 4, 409 (1998); OPAL Collaboration, K. Ackerstaff et al., ibid. 7, 571 (1999).

[4] B. Chibishov et al., Int. J. Mod. Phys. A 12, 2075 (1997); V. A. Novikov et al., Nucl. Phys. B237, 525 (1984).

[5] S. J. Brodsky et al., Phys. Lett. B 372, 133 (1996).

[6] S. J. Brodsky and H. J. Lu, Phys. Rev. D 51, 3652 (1995).

[7] G. Grunberg, Phys. Rev. D 29, 2315 (1984).

[8] S. G. Girishny, A. L. Kataev, and S. A. Larin, Phys. Lett. B 259, 144 (1991).

[9] E. C. Poggio, H. R. Quinn, and S. Weinberg, Phys. Rev. D 13, 1958 (1976).

[10] T. W. Appelquist and H. D. Politzer, Phys. Rev. Lett. 34, 43 (1975); Phys. Rev. D 12, 1404 (1975).
[11] J. Schwinger, Particles, Sources and Fields, Vol. II (AddisonWesley, New York, 1973).

[12] S. Brodsky et al., Phys. Rev. D 58, 116006 (1998); A. H. Hoang, Z. Ligeti, and A. V. Manohar, ibid. 59, 074017 (1999).

[13] A. C. Mattingly and P. M. Stevenson, Phys. Rev. D 49, 437 (1994).

[14] A. Quenzer, Phys. Lett. 76B, 512 (1978); J. Burmeister et al., ibid. 76B, 361 (1978); Ch. Berger et al., ibid. 81B, 410 (1979); C. Bacci et al., ibid. 86B, 234 (1979); J. L. Siegrist et al., Phys. Rev. D 26, 969 (1982); B. Niczyporuk et al., Z. Phys. C 15, 299 (1982); L. M. Barkov et al., Nucl. Phys. B256, 365 (1985); Z. Jakubobski et al., Z. Phys. C 40, 49 (1988); D. Bisello et al., Phys. Lett. B 220, 325 (1989); W. Bartel et al., Phys. Lett. 129B, 145 (1983); 160B, 337 (1985); B. Naroska et al., Phys. Rep. 148, 67 (1987); B. Adeva et al., Phys. Rev. D 34, 681 (1986); R. Brandelik et al., Phys. Lett. 113B, 499 (1982); M. Althoff et al., ibid. 138B, 441 (1984); H.-J. Behrend et al., Phys. Lett. B 183, 407 (1987).

[15] M. L. Swartz, Phys. Rev. D 53, 5268 (1996).

[16] V. A. Novikov et al., Phys. Rep. 41, 1 (1978); M. B. Voloshin, Int. J. Mod. Phys. A 10, 2865 (1995); S. J. Brodsky et al., Phys. Lett. B 359, 355 (1995). 